\title{
Editorial
}

\section{Heterogeneous Photochemistry: Solar Energy Conversion and Environmental Remediation}

\author{
Daniele Dondi, ${ }^{1}$ Sandra Babić, ${ }^{2}$ Irene Michael, ${ }^{3}$ Giovanni Palmisano, ${ }^{4}$ and Andrea Speltini ${ }^{1}$ \\ ${ }^{1}$ Department of Chemistry, University of Pavia, Viale Taramelli 12, 27100 Pavia, Italy \\ ${ }^{2}$ Department of Analytical Chemistry, University of Zagreb, Marulicev Trg 20, 10000 Zagreb, Croatia \\ ${ }^{3}$ Department of Civil and Environmental Engineering, University of Cyprus, Kallipoleos Street 75, 1678 Nicosia, Cyprus \\ ${ }^{4}$ Department of Chemical and Environmental Engineering, Institute Center for Water and Environment (iWater), \\ Masdar Institute of Science and Technology, P.O. Box 54224, Abu Dhabi, UAE \\ Correspondence should be addressed to Daniele Dondi; daniele.dondi@unipv.it
}

Received 14 July 2016; Accepted 14 July 2016

Copyright (C) 2016 Daniele Dondi et al. This is an open access article distributed under the Creative Commons Attribution License, which permits unrestricted use, distribution, and reproduction in any medium, provided the original work is properly cited.

This special issue contains original research articles on solar energy use for green applications. In particular, the use of solar thermal energy for low environmental impact processes or the use of the light (with or without heterogeneous photocatalysts) for environmental remediation or synthesis was considered. The topic is highly challenging and the subject would be important, timely, and of great interest. Among the submitted manuscripts, 5 were selected as suitable for publication through the strict experts' peer reviews.

The papers to be published in this special issue are "Design and Performance Evaluation of a Solar Assisted Heat Pump Dryer Integrated with Biomass Furnace for Red Chilli" for the direct solar light/thermal applications; "Sorbents Coupled to Solar Light $\mathrm{TiO}_{2}$-Based Photocatalysts for Olive Mill Wastewater Treatment," "Photodegradation of 2,4Dichlorophenol in Aqueous Systems under Simulated and Natural Sunlight," and "Flexible Bench-Scale Recirculating Flow CPC Photoreactor for Solar Photocatalytic Degradation of Methylene Blue Using Removable $\mathrm{TiO}_{2}$ Immobilized on PET Sheets" for the photodegradation topic; and "Noble Metal Decoration and Presulfation on $\mathrm{TiO}_{2}$ : Increased Photocatalytic Activity and Efficient Esterification of n-Butanol with Citric Acid" for synthetic photochemistry.

We believe that this special issue can be interesting and inspiring for the readers of the journal since this advanced interdisciplinary technology involves a wide range of subjects.

Daniele Dondi Sandra Babić

Irene Michael Giovanni Palmisano Andrea Speltini 

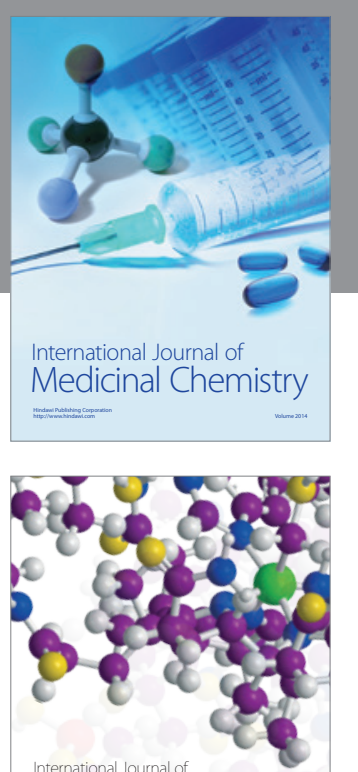

Carbohydrate Chemistry

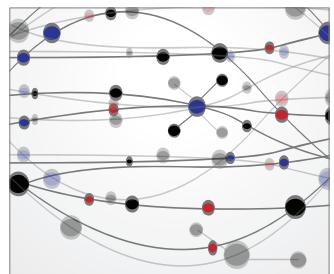

The Scientific World Journal
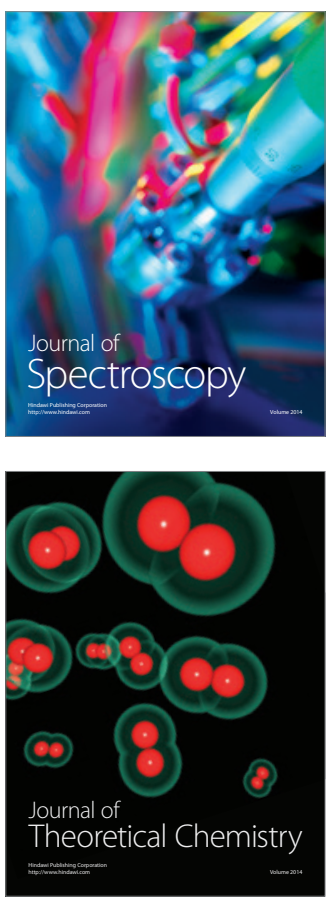
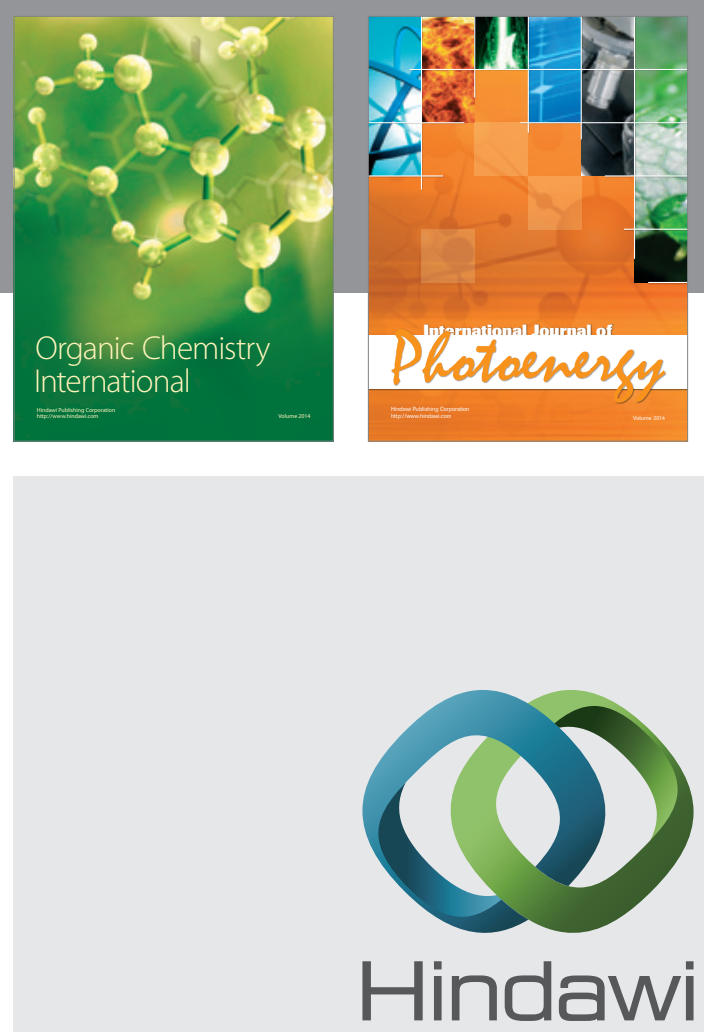

Submit your manuscripts at

http://www.hindawi.com

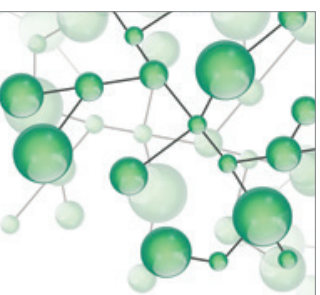

International Journal of

Inorganic Chemistry

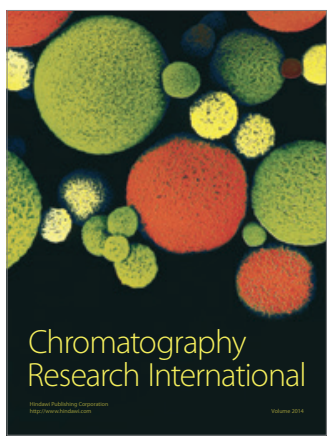

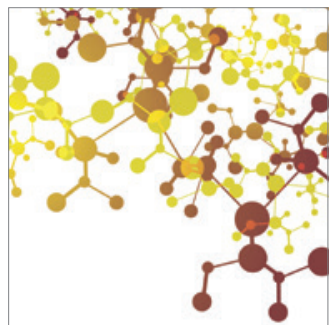

Applied Chemistry
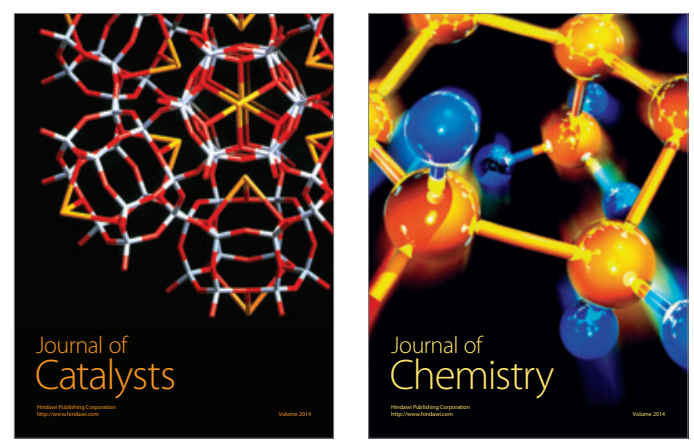
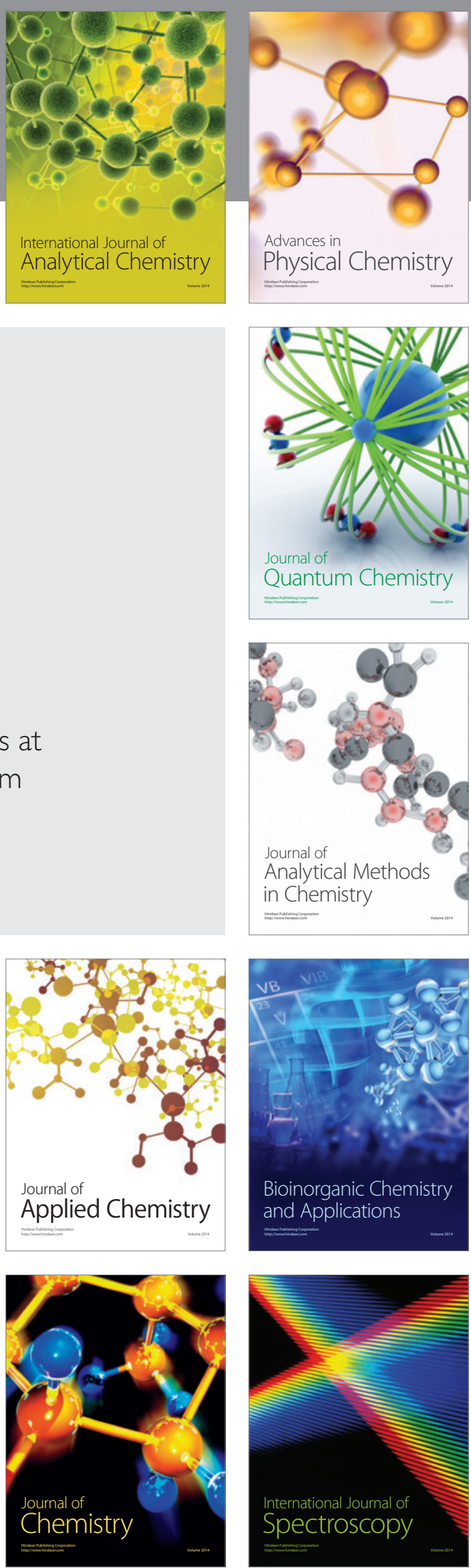